\title{
Prospects of Circular Electron Positron Collider Construction
}

\author{
Xinming Shen \\ Independent Schools Foundation, Shenzhen, 518000,China, 4120001@student.isf.edu.hk
}

\begin{abstract}
The Circular Electron Positron Collider (CEPC) is an advanced accelerator designed to investigate the limitations of $\mathrm{SM}$, and it is still in the planning stage. The paper summarized the history of CEPC planning, current development, and prospect of CEPC.
\end{abstract}

Keywords: CEPC, High Energy Physics.

\section{THE INTRODUCTION}

Every breakthrough in the Standard Model is based on the results of high-energy particle experiments. However, only a country has the money and manpower to build a high-energy particle experiment at this scale. The world's most powerful particle collider is Large Hadron Collider (LHC) at CERN, which has a $27 \mathrm{~km}$ circumference and $14 \mathrm{TeV}$ of energy and costs $\$ 8$ billion. Various experiments at LHC show that almost no violation of the Standard Model is found at energy scales below $\mathrm{TeV}$ magnitude.

The Higgs bosons are particles predicted by the Standard Model. They have no electric charge, no color charge and are unstable. In 1964, British scientist Peter Higgs proposed the existence of the Higgs field in order to explain the origin of "mass," and then predicted the existence of the Higgs bosons.

In 2012, the European Organization for Nuclear Research (CERN) announced that the Higgs boson was found at the Large Hadron Collider (LHC) at the CMS and ATLAS detectors, respectively, with a level of certainty of 5 sigma and a mass of about $125 \mathrm{GeV}$. From a statistical point of view, it can be assumed that the particle has been discovered. It is a very important breakthrough for particle physics.

To date, the LHC has produced tens of millions of Higgs bosons at the record-breaking collision energy of $13 \mathrm{TeV}$. Its further upgrade project "HL-
LHC" is aiming for more. However, due to the very large proton-proton cross-section at LHC, the background noise is relatively high, resulting in that many Higgs Bosons generated cannot be recorded. At the same time, protons are not elementary particles but complex systems of quark and gluons, so some errors are inevitable due to the complexity of systems. These disadvantages make it difficult for the LHC to make accurate measurements of Higgs particle properties [1].

According to the Higgs mechanism, the Higgs Bosons interact with elementary particles to give them mass. So, it may also interact with undiscovered particles. And since the mass of the Higgs boson is only $125 \mathrm{GeV}$, the threshold production energy is similar to the maximum energy of the existing LHC. If a collider can be built with higher energy and brightness, it will be possible to study Higgs Bosons in a more accurate way.

\section{THE HISTORY PLANNING}

Under those circumstances, in 2012, highenergy physicists in China proposed to build a collider, known as Circular Electron Positron Collider (CERN) later. CERN then included the proposal as part of its five-year plan for 2013-2018 and launched research on the design of the collider in 2014. The International Committee for the Future Accelerator (ICFA) has officially announced that it will encourage global collaboration. In the future, the Super Proton-Collider (SppC) with a center of 
mass energy of 50-100 $\mathrm{TeV}$ can be built in the same tunnel.

At present, after many discussions, some details of the CEPC have been finalized. The main ring of CPEC is $100 \mathrm{~km}$ in circumference (nearly four times larger than that of LHC). Figure 2.1 shows its engineering design. It is planned to be built in Shanwei city. At a center energy of $240 \mathrm{GeV}, \mathrm{CPEC}$ is expected to produce a million Higgs Bosons, nearly six times more than the ILC, which creates a huge progress in precision. CPEC is also expected to produce $\mathrm{W}$ bosons and $\mathrm{Z}$ bosons at very low background noise level. At the same time, CEPC can be used for QCD measurement. In short, CEPC enables accurate measurement and verification of the Standard Model in all aspects, leading to the exploration of new physics [2].

CEPC has huge advantages in the production of Higgs bosons. Electrons and positrons are the basic particles of the Standard Model, which means that the initial state of the collider is precisely known (compared to protons of LHC). One Higgs particle can be created every 100-1000 electron-positron collisions, with a much smaller background noise than the LHC's. With the support of advanced detector systems, almost all signal instances can be recorded. In addition, it allows accurate, independent measurements of Higgs bosons' properties.

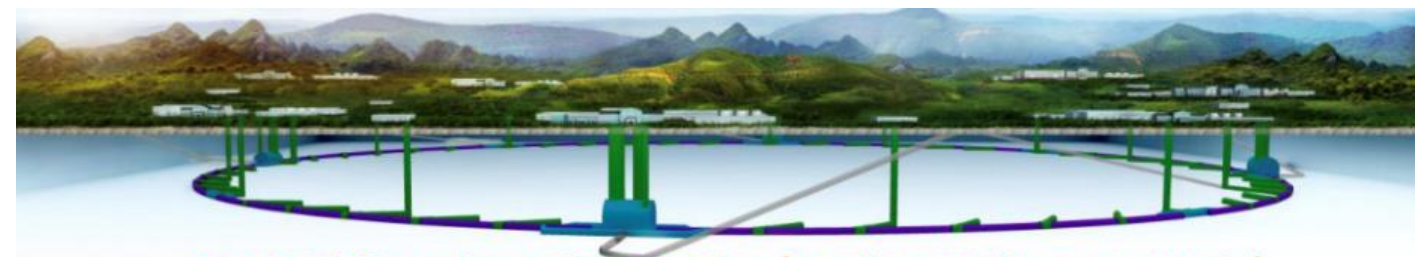

CEPC Civil Enginnering Design (Funing $100 \mathrm{~km}$, example)

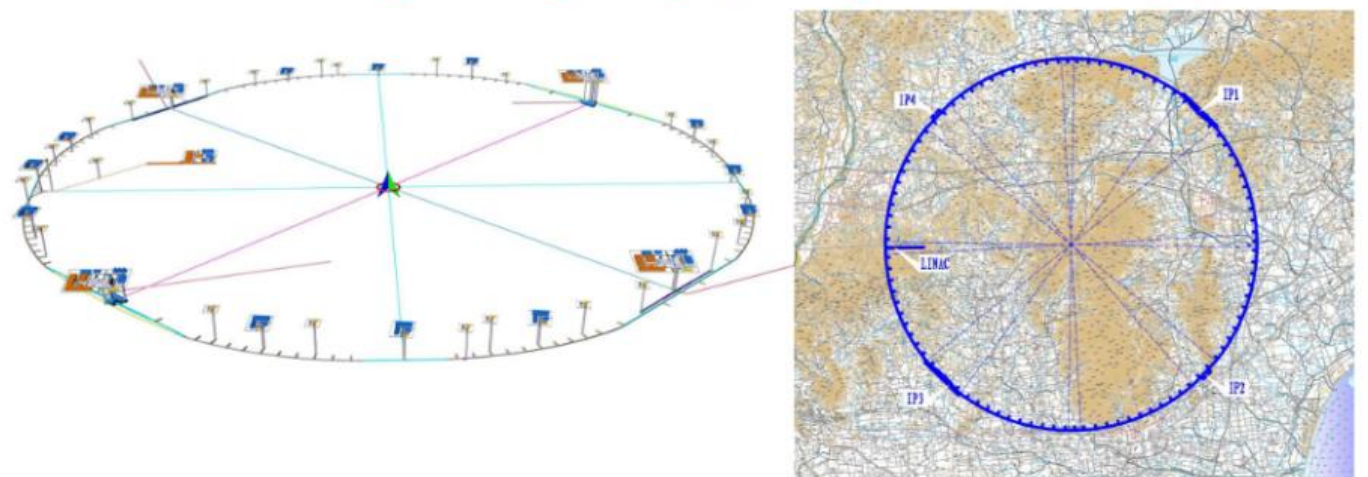

Figure 1 The CEPC Engineering Design Graph [3]

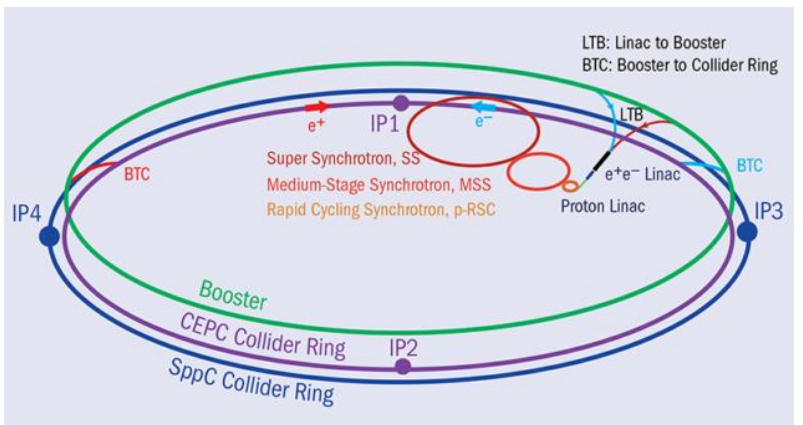

Figure 2 The Overall CEPC Schematic Layout [4]

\section{THE DEVELOPMENT}

After the discovery of the Higgs boson in 2012, the collider attracted increasing attention around the world, and China was no exception. In September, the CEPC-SppC construction plan was first proposed by the Institute of High-Energy Physics (IHEP), Chinese Academy of Sciences.
In 2015, the first international review meeting of CEPC was held by IHEP. The "Preliminary Conceptual Design Report (Pre-CDR)" was released, including Volume I (Physics and Detector) and Volume II (Accelerator), shown in Figure 3.

The report confirmed the feasibility of the CEPC. It suggests that the CEPC project does not face fundamental difficulties, and it identifies a number of key technologies that require further research. According to the report, the CEPC working group has carried out a large number of scientific research and completed the preliminary research on a series of key technologies on the collider and detector (such as identifying that the key technologies of CEPC are superconducting radio frequency devices) [5]. 


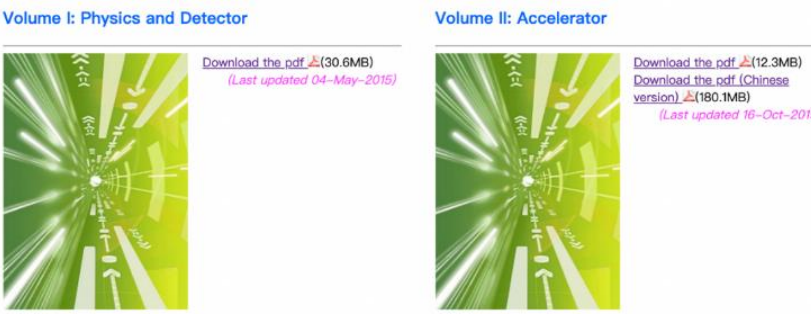

Figure 3 Preliminary Conceptual Design Report (PreCDR), Volume I and II

In 2016, “Announcement of Bid Invitation for Civil Construction of CEPC-SppC" was released by IHEP. It includes a construction overview and possible sites [6].

On November 14, 2018, two volumes of Conceptual Design Report were officially released. They describe the baseline design and technical details of the accelerator and the detector of CEPC. The scientific advantages of CEPC over the Large Hadron Collider (LHC) are also evaluated in detail. The reports are very detailed and comprehensive, "[it] contains the work of thousands of scientists over the past six years." (The CEPC Study Group, 2018).

The Conceptual Design Report contains Volume I-Accelerator and Volume II-Physics \& Detector. It gives baseline designs for the CEPC collider (Figure 2.2.2), which means they now have a collider-detector design that works in theory.

The Accelerator Volume introduces the whole accelerator design, including collider accelerator, booster accelerator, linear damping, and systems. In addition, a series of important support facilities such as cryogenic system or radiation protection are also included, and possible options for upgrading CEPC are discussed. The Detectors and Physics Volume explains the aim of CEPC, presents the experiment conditions, introduces the design of components such as calorimetry and their key technology systems, focuses on an in-depth evaluation of detector and physics research in CEPC, and discusses future development and research direction $[7,8]$. On November, a meeting on key technologies of CEPC was held in IHEP.

The CEPC project team plans to complete key technology research based on the Concept Design
Report and plans to build a series of prototypes of key components before 2022. According to the current schedule, CEPC will complete the technical design report and test those technologies in an industrial scale in about five years, which then means that the technologies of the CEPC project are perfected and the actual construction can be started.

\section{THE PROSPECTS}

CEPC is a big goal of particle physicists in China. Once it is built, China will undoubtedly become one of the world leaders in high-energy collider physics. However, because of the huge size of CPEC, it requires national and even international support.

The pre-research, construction, and operation of a huge particle collider usually take about 20-30 years. Moreover, the construction and operation cost a lot, which requires not only financial support from the national level, but also financial support from international cooperation.

Wang Yifang, academician of the Chinese Academy of Sciences and director of the IHEP, said in her speech "High-Energy Physics in China: Past, Present and Future" that the cost of CPEC is about 40 billion Yuan, which is only $0.05 \%$ of China's GDP. In contrast, there are argument from other physicists. Academician Yang Zhenning is very opposed to the construction of CEPC. He argued that the 40 billion yuan is just the cost of the project alone, not including the costs of operation, maintenance and scientific research. After all, China is still a developing country and should not spend too much money on such a project with no clear guarantee of outcome. At present, whether China should build CEPC is still controversial.

Now, CEPC already has a conceptual design, but it still needs a long time to complete technical design, and its technical difficulties need to be fully explored. The estimate of the cost and operation cost of CEPC also needs to be further refined, so as to reevaluate the cost and value of its construction. At present, the time planning for CEPC is shown in Table 2. Note that some plans have been postponed because of the outbreak of coronavirus. 
Table 1 The CEPC Parameters [7]

\begin{tabular}{|c|c|c|c|c|}
\hline & Higgs & $\mathrm{W}$ & $\mathrm{Z}(3 \mathrm{~T})$ & $\mathrm{Z}(2 \mathrm{~T})$ \\
\hline Number of IPs & \multicolumn{4}{|c|}{2} \\
\hline Beam Energy $(\mathrm{GeV})$ & 120 & 80 & & \\
\hline Circumference $(\mathrm{km})$ & \multicolumn{4}{|c|}{100} \\
\hline $\begin{array}{l}\text { Synchrotron radiation } \\
\text { loss/turn }(\mathrm{GeV})\end{array}$ & 1.73 & 0.34 & \multicolumn{2}{|c|}{0.036} \\
\hline Crossing angle at IP (mrad) & \multicolumn{4}{|c|}{$16.5 \times 2$} \\
\hline Piwinki angle & 3.48 & 7.0 & \multicolumn{2}{|c|}{23.8} \\
\hline Particles/bunch $N_{e}\left(10^{10}\right)$ & 15.0 & 12.0 & \multicolumn{2}{|c|}{8.0} \\
\hline Bunch number & 242 & 1524 & \multicolumn{2}{|c|}{$12000(10 \%$ gap $)$} \\
\hline Bunch spacing (ns) & 680 & 210 & \multicolumn{2}{|c|}{25} \\
\hline Beam current (mA) & 17.4 & 87.9 & \multicolumn{2}{|c|}{461.0} \\
\hline Synch. Radiation power(MW) & 30 & 30 & \multicolumn{2}{|c|}{16.5} \\
\hline Bending radius $(\mathrm{km})$ & \multicolumn{4}{|c|}{10.7} \\
\hline Momentum compaction $\left(10^{-5}\right)$ & \multicolumn{4}{|c|}{1.11} \\
\hline
\end{tabular}

In the future, technical difficulties of both foreign and domestic collider construction will be of international level, so international cooperation is inevitable. Many of the specific engineering details and technologies of CEPC have been implemented on LHC, so a lot of international reference and cooperation may be used to build the CEPC. For example, many overseas professors and researchers have participated in the CPEC Conceptual Design Report. Only by actively participating in the international cooperation can the CEPC project be fully supported by the international high-physics community. [9] [10]

Table 2 The Construction Plan for CEPC-SppC

\begin{tabular}{|c|c|}
\hline Years & Plan \\
\hline $2015-2020$ & CEPC Conceptual Design \\
\hline $2021-2027$ & CEPC Construction \\
\hline $2027-2035$ & CEPC Operation \\
\hline $2015-2030$ & SppC Conceptual Design \\
\hline $2030-2040$ & SppC Construction \\
\hline $2040-2050$ & SppC Operation \\
\hline
\end{tabular}

\section{CONCLUSION}

The CPEC construction plan has gone through a lot of detailed refinements, and the parameters and precision have been improved. At present, the construction of CEPC is still under heated discussion. [11] Whether China should spend so much money to build it is still being discussed by government officers and scientists. What is certain, however, is that the construction of accelerators such as CEPC will play a crucial role in the next evolution of particle physics. If constructed, CEPC will become an international research center for high-energy physics. This has led to further exploration of the Standard Model, of high-energy physics, and of the nature of the universe.

\section{REFERENCES}

[1] Facts and figures about the LHC | CERN. (2021, April 14). Home.cern. https://home.cern/resources/faqs/facts-andfigures-about-lhc

[2] CEPC. (2021). Ihep.ac.cn. http://cepc.ihep.ac.cn/

[3] Overview of Completion of CEPC Accelerator. (2018). https://indico.ihep.ac.cn/event/8299/session $/ 2 /$ contribution $/ 2 /$ material/slides $/ 0$.pdf

[4] China's bid for a circular electron-positron collider - CERN Courier. (2018, June). CERN

Courier.

https://cerncourier.com/a/chinas-bid-for-acircular-electron-positron-collider/

[5] CEPC-SppC Preliminary Conceptual Design Report. (2015). Ihep.ac.cn. http://cepc.ihep.ac.cn/preCDR/volume.html

[6] Announcement of Bid Invitation for Civil Construction of CEPC-SppC. (2016). Ihep.cas.cn.

http://www.ihep.cas.cn/xwdt/tzgg/201601/t 20160113_4517708.html

[7] CEPC Conceptual Design Report Volume I Accelerator. (2018, January). http://cepc.ihep.ac.cn/CEPC_CDR_Vol1_A ccelerator.pdf

[8] CEPC Conceptual Design Report Volume II Physics \& Detector. (2018, January). http://cepc.ihep.ac.cn/CEPC_CDR_Vol2_P hysics-Detector.pdf

[9] The Next Generation High-Energy ElectronPositron Collider: Status Quo and Countermeasures. (2014). Ihep.cas.cn. http://www.ihep.cas.cn/kxcb/kjqy/201406/t 20140610_4134166.html 
[10] Elementary Particle. (2011, January 5). Science.

Online. https://highscope.ch.ntu.edu.tw/wordpress/? $\mathrm{p}=19087$
[11] Institute of High Energy Physics, Chinese Academy of Sciences. (2017). National Science Review, 4(6), 934-942. https://doi.org/10.1093/nsr/nwx141 